\title{
Identification of Tetrabromobisphenol A Allyl Ether and Tetrabromobisphenol A 2,3-Dibromopropyl Ether in the Ambient Environment near a Manufacturing Site and in Mollusks at a Coastal Region
}

\author{
Guangbo Qu, Aifeng Liu, Thanh Wang, Chaoli Zhang, Jianjie Fu, Miao Yu, Jianteng Sun, Nali Zhu,
} Zhuona Li, Guohua Wei, Yuguo Du, Jianbo Shi,* Sijin Liu,* and Guibin Jiang

State Key Laboratory of Environmental Chemistry and Ecotoxicology, Research Center for Eco-Environmental Sciences, Chinese Academy of Sciences, Beijing 100085, People's Republic of China

\section{Supporting Information}

ABSTRACT: Tetrabromobisphenol A (TBBPA) is one of the most widely used brominated flame retardants (BFRs) and has been frequently detected in the environment and biota. Recent studies have found that derivatives of TBBPA, such as TBBPA bis(allyl) ether (TBBPA BAE) and TBBPA bis(2,3dibromopropyl) ether (TBBPA BDBPE) are present in various environmental compartments. In this work, using liquid chromatography coupled with tandem mass spectrometry (LC-MS/MS) and liquid chromatography coupled with quadrupole time-of-flight mass spectrometry (LC-Q-TOF-MS), TBBPA allyl ether (TBBPA AE) and TBBPA 2,3-dibromopropyl ether (TBBPA DBPE) were identified in environmental samples and further confirmed by synthesized standards. Soil, sediment, rice hull, and earthworm samples collected near a BFR manufacturing plant were found to contain these two compounds. In sediments, the concentrations of TBBPA AE and TBBPA DBPE ranged from 1.0 to $346.6 \mathrm{ng} / \mathrm{g}$ of dry weight $(\mathrm{dw})$ and from 0.7 to $292.7 \mathrm{ng} / \mathrm{g}$ of $\mathrm{dw}$, respectively. TBBPA AE and TBBPA DBPE in earthworm and rice hull samples were similar to soil samples, which ranged from below the method limit of detection (LOD, $<0.002 \mathrm{ng} / \mathrm{g}$ of $\mathrm{dw}$ ) to $0.064 \mathrm{ng} / \mathrm{g}$ of dw and from below the LOD $(<0.008 \mathrm{ng} / \mathrm{g}$ of $\mathrm{dw}$ ) to $0.58 \mathrm{ng} / \mathrm{g}$ of $\mathrm{dw}$, respectively. Furthermore, mollusks collected from the Chinese Bohai Sea were used as a bioindicator to investigate the occurrence and distribution of these compounds in the coastal environment. The detection frequencies of TBBPA $\mathrm{AE}$ and TBBPA DBPE were 41 and 32\%, respectively, and the concentrations ranged from below $\mathrm{LOD}(<0.003 \mathrm{ng} / \mathrm{g}$ of $\mathrm{dw})$ to $0.54 \mathrm{ng} / \mathrm{g}$ of $\mathrm{dw}$, with an average of $0.09 \mathrm{ng} / \mathrm{g}$ of $\mathrm{dw}$, for TBBPA AE, and from below LOD (<0.008 ng/g of dw) to $1.41 \mathrm{ng} / \mathrm{g}$ of $\mathrm{dw}$, with an average of $0.15 \mathrm{ng} / \mathrm{g}$ of $\mathrm{dw}$, for TBBPA DBPE.

\section{INTRODUCTION}

Concerns have recently been raised for some brominated flame retardants (BFRs) because of their persistence in the environment, widespread global distribution, and potential adverse health effects. ${ }^{1-3}$ In addition to the potential adverse effects of some BFRs, their byproducts and transformation products might also be of concern. ${ }^{4-8}$ The general evaluation of the effects of BFRs on the environment and human health might necessitate the identification of previously unknown brominated substances in the environment and byproducts or transformation products of some main BFRs. ${ }^{4,7}$

Tetrabromobisphenol A (TBBPA) is currently considered the most widely used $B F R,{ }^{4,9}$ with its usage exceeding those of decabromodiphenyl ether (decaBDE) and hexabromocyclododecane (HBCD). ${ }^{10}$ TBBPA could be modified to yield TBBPA derivatives, such as TBBPA bis(allyl) ether (TBBPA BAE) and TBBPA bis(2,3-dibromopropyl) ether (TBBPA BDBPE) via modification of the TBBPA hydroxyl groups. ${ }^{4,10}$ TBBPA derivatives are used in polymer products or as precursor

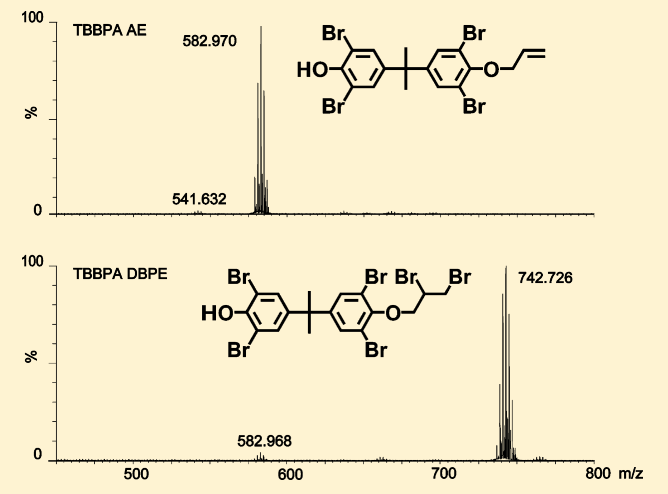


coupled with tandem mass spectrometry (HPLC-MS/MS) and gas chromatography coupled with mass spectrometry (GC-MS) have been previously used to analyze some TBBPA derivatives. $^{11,12}$ In a previous study on TBBPA BAE and TBBPA BDBPE, Letcher et al. applied HPLC coupled with atmospheric pressure photoionization (APPI) MS/MS as the analysis method. ${ }^{13,15}$ A HPLC coupled with electrospray ionization (ESI) MS/MS method was also applied to determine TBBPA BAE in lake trout. ${ }^{16}$ TBBPA BAE and TBBPA BDBPE was found in herring gull eggs near the Great Lakes, which suggested that these BFRs are potentially bioaccumulative and persistent in the environment. ${ }^{13} \mathrm{Qu}$ et al. further found that TBBPA BAE was probably the main causative chemical for the neurotoxic fraction in some environmental samples near a BFR manufacturing facility. ${ }^{15}$ In 2006 , the production volume of TBBPA was approximately 150000 tons/year worldwide. ${ }^{17}$ The production amount of TBBPA BAE in the U.S. was 230 tons. ${ }^{8}$ Although the production volume of TBBPA BAE is not clear in China, it is currently produced in factories localized in several provinces, such as Hunan, Shandong, Jiangsu, Zhejiang, Guangdong, Jiangsu, and Shanghai (http://www.chemicalbook. com). For TBBPA BDBPE, the production amount in China and the U.S. in 2006 was 4000 and 4500 tons, respectively. ${ }^{4,18,19}$ Because these BFRs could be produced and used in large quantities, they may also be further released into the environment. Nevertheless, there are currently no literature report data on the potential transformation product or byproduct of TBBPA BAE and TBBPA BDBPE in the environment.

We hypothesize that TBBPA allyl ether (TBBPA AE) and TBBPA 2,3-dibromopropyl ether (TBBPA DBPE), as the possible byproduct or transformation product of TBBPA derivatives, could also be formed during TBBPA BAE and TBBPA BDBPE production and application. TBBPA derivatives are synthesized using TBBPA as raw material, and TBBPA AE and TBBPA DBPE can also be unintentionally produced as a result of the incomplete formation of both ether bonds leaving one unmodified hydroxyl group. Furthermore, TBBPA has been considered as a possible degradation product of TBBPA BAE and TBBPA BDBPE because of the breaking of the ether bonds. ${ }^{16}$ This reaction could also produce intermediate products, such as TBBPA AE and TBBPA DBPE.

In this study, we aimed to investigate the occurrence and distribution of TBBPA, TBBPA BAE, and TBBPA BDBPE and their analogues TBBPA AE and TBBPA DBPE. Because no commercial standards are available for TBBPA AE and TBBPA DBPE, we synthesized and characterized these two TBBPA derivatives and also developed a sensitive HPLC-MS/MS method for their identification and quantification.

We analyzed various environmental matrices near a BFR manufacturing plant and also mollusks around the coastal area of northeastern China for these compounds. To the best of our knowledge, this is the first study to report the mass spectrum and develop a sensitive analytical method for the determination of TBBPA AE and TBBPA DBPE and also their presence in the environment.

\section{MATERIALS AND METHODS}

Chemicals. TBBPA (purity of 99.8\%) was purchased from AccuStandard (New Haven, CT) and used as an analytical standard. TBPPA (purity of 97\%) purchased from Alfa Aesar (Ward Hill, MA) was used for TBBPA AE and TBBPA DBPE synthesis. TBBPA BAE (purity of 98.9\%) and TBBPA BDBPE (purity of 97.5\%) were purchased from Sigma-Aldrich (St. Louis, MO).

Instrumentation. HPLC coupled with quadrupole time-offlight mass spectrometry (HPLC-Q-TOF-MS, Waters, Milford, MA) was used for chemical identification. Waters 2695 HPLC (Waters, Milford, MA) either coupled with an ultraviolet-visible (UV-vis) absorbance detector (2487 IEEE, Waters, Milford, MA), Quattro Ultima triple quadrupole mass spectrometer (Waters, Milford, MA), or atmospheric pressure chemical ionization (APCI) or ESI source (Waters, Milford, MA) (HPLC-APCI/ESI-MS/MS) was used to determine the target compounds in environmental matrices. The system was controlled by the Masslynx 4.1 software (Waters, Milford, MA). An automatic gel permeation chromatography instrument (GPC, AccuPrep, J2-Scientific; Bio-Beads S-X3) was used for primary cleanup.

Sample Collection. In 2010, agricultural soil and surface sediment samples were collected up- and downstream of the effluent outlet of a BFR factory at Liuyang River in Liuyang, Hunan, China. The sampling sites are shown in Figure S1 of the Supporting Information. Site A is located $0.5 \mathrm{~km}$ upstream of the plant outlet, and sites B, C, D, E, and F are located 0.5, $3.1,5,6.5$, and $7.7 \mathrm{~km}$ downstream of the plant outlet, respectively (see Figure S1 of the Supporting Information). Rice hull has been reported to adsorb various organic contaminants because of its unique surface structure and could also be used as an indicator medium for the contamination of persistent organic pollutants (POPs). ${ }^{20}$ Also, earthworm has been used as a bioindicator for organic contaminants and could reflect the potential bioavailability of chemicals, such as BFRs. ${ }^{21}$ Thus, these samples were also collected at sites B, C, D, and E for further analysis. All of the solid samples were freeze-dried, and concentrations were reported on a dry weight $(\mathrm{dw})$ basis.

The Bohai Sea in China is a semi-enclosed gulf in northeastern China and surrounded by four provinces, including a number of large cities near its shoreline. About $18 \%$ of the total population of China lives in this region, which is an important economic rim that contributes to $28 \%$ of the total gross domestic product (GDP). Mollusks are invertebrate benthic organisms inhabiting marine and freshwater environments with a wide geographical distribution. These have often been used as ideal bioindicators of pollution because of their sessile lifestyle and potential to accumulate organic pollutants. ${ }^{22,23}$ In 2010, 11 species of mollusks were collected from nine cities (Figure S1 of the Supporting Information): Dalian (DL), Yingkou (YK), Huludao (HLD), Beidaihe (BDH), Tianjin (TJ), Shouguang (SG), Penglai (PL), Yantai (YT), and Weihai (WH). The 11 selected species of mollusks are Neverita didyma (Nev), Rapana venosa (Rap), Mya arenaria (Mya), Cyclina sinensis (Cyc), Chlamys farreri (Chl), Scapharca subcrenata (Sca), Meretrix meretrix (Mer), Mytilus edulis (Myt), Crassostrea talienwhanensis (Ost), Amusium (Amu), and Mactra veneriformis (Mac). The collected mollusks were transported to the laboratory on ice. After excision by stainlesssteel scalpel blades, the soft tissue of the mollusks was thoroughly rinsed with Milli-Q water to remove extraneous impurities. For each species from a sampling site, about 500$1500 \mathrm{~g}$ of wet soft tissue was homogenized in a blender to form one composite sample. The samples were kept at $-20{ }^{\circ} \mathrm{C}$ until analysis. A total of 84 composite soft tissue samples were analyzed in this study. All of the mollusk samples were freezedried, and concentrations were reported on a dw basis. 
TBBPA AE Synthesis. TBBPA AE was synthesized according to the scheme shown in Figure S2 of the Supporting Information. To a solution of TBBPA (Alfa Aesar, Ward Hill, MA) $(1.9 \mathrm{~g})$ and allyl bromide $(0.3 \mathrm{~mL})$ in acetone $(10 \mathrm{~mL})$, sodium hydroxide ( $0.28 \mathrm{~g}$ in $2 \mathrm{~mL}$ of water) was added at room temperature. The reaction mixture was stirred for $2 \mathrm{~h}$. The reaction mixture was diluted with dichloromethane (DCM) and washed with water. The organic layer was dried over $\mathrm{MgSO}_{4}$. After removal of the solvent, the residue was purified by column chromatography on silica gel, yielding TBBPA AE.

TBBPA DBPE Synthesis. The synthesis scheme for TBBPA DBPE is also seen in Figure S2 of the Supporting Information. To a solution of TBBPA AE $(1 \mathrm{~g})$ in carbon tetrachloride (10 $\mathrm{mL})$, bromine $(0.175 \mathrm{~mL})$ was added at room temperature. The reaction mixture was stirred for $1 \mathrm{~h}$. The reaction mixture was diluted with DCM and washed in sequence with saturated sodium thiosulfate, saturated sodium bicarbonate, and water. The organic layer was dried over $\mathrm{MgSO}_{4}$. After removal of the solvent, the residue was purified by column chromatography on silica gel, giving TBBPA DBPE.

Instrumental Parameters of Q-TOF-MS. For identification of TBBPA AE and TBBPA DBPE, the synthesized standards were analyzed using HPLC-Q-TOF-MS at full-scan mode from $\mathrm{m} / \mathrm{z} 450$ to 800 under ESI mode. The parameters for the mass spectrometer are summarized as follows: corona current, $10 \mu \mathrm{A}$; source temperature, $120{ }^{\circ} \mathrm{C}$; probe temperature, $630{ }^{\circ} \mathrm{C}$; cone gas flow, $40 \mathrm{~L} / \mathrm{h}$; and desolvation gas flow, $100 \mathrm{~L} / \mathrm{h}$.

Instrumental Parameters of HPLC-MS/MS and HPLC-UV. For HPLC-APCI-MS/MS optimization, individual standard solutions of TBBPA (purity of 99.8\%, AccuStandard), TBBPA AE (synthesized, purity of 98.0\%), TBBPA DBPE (synthesized, purity of 97.9\%), TBBPA BAE (Sigma-Aldrich, purity of 98.9\%), and TBBPA BDBPE (SigmaAldrich, purity of $97.5 \%$ ) were injected to obtain mass spectra. APCI was used under full-scan mode to generate the precursor ions, which were then isolated by the first quadrupole. Full-scan data acquisition was performed by scanning from $\mathrm{m} / \mathrm{z} 50$ to 1000 , with a scan time of $1 \mathrm{~s}$, step size of 0.1 atomic mass unit (amu), and an interscan delay of $5 \mathrm{~ms}$. To select product ions, under multiple reaction monitoring (MRM) mode, the selected precursors were broken in the second quadrupole as a collision cell to yield product ions, which were scanned in the third quadrupole. The collision energy was optimized manually, and product ion scan mass spectra were recorded. The optimized parameters for the mass spectrometer are summarized as follows: corona current, $5 \mu \mathrm{A}$; source temperature, $120{ }^{\circ} \mathrm{C}$; probe temperature, $630{ }^{\circ} \mathrm{C}$; cone gas flow, $40 \mathrm{~L} / \mathrm{h}$; and desolvation gas flow, $1000 \mathrm{~L} / \mathrm{h}$. MRM was performed using a dwell time of $200 \mathrm{~ms}$ for the ionization methods. The cone voltage and collision energy are shown in Table S1 of the Supporting Information. Suitable precursor ions were identified and selected from MS scan mode. After optimization, the C18 column $(150 \times 4.6 \mathrm{~mm}, 5 \mu \mathrm{m}$ particle size, Sunfire $)$ was used with an initial mobile phase setting of $90 \%$ methanol (A) and $10 \%$ water (B) and a linear gradient elution from 90 to $100 \%$ A over a $10 \mathrm{~min}$ period, followed by an isocratic hold for $5 \mathrm{~min}$ at $100 \% \mathrm{~A}$.

The HPLC-ESI-MS/MS method was optimized under negative-ion mode with the following optimized parameters: capillary voltage, $2.5 \mathrm{kV}$; source and desolvation temperature, 120 and $450{ }^{\circ} \mathrm{C}$, respectively; desolvation gas flow, $450 \mathrm{~L} / \mathrm{h}$; and cone gas flow, $50 \mathrm{~L} / \mathrm{h}$. Argon gas pressure in the collision cell was kept at $3.3 \times 10^{-3}$ mbar for MS/MS measurements. MRM was performed using a dwell time of $200 \mathrm{~ms}$ for the ionization methods. The cone voltage and collision energy are shown in Table $\mathrm{S} 1$ of the Supporting Information. After optimization, a C18 column $(150 \times 2.1 \mathrm{~mm}, 5 \mu \mathrm{m}$ particle size, Sunfire) was selected for the separation of target compounds. HPLC separation was carried out with an initial mobile phase setting of $90 \%$ methanol (A) and $10 \%$ water (B) at a flow rate of $0.3 \mathrm{~mL} / \mathrm{min}$, increased to $100 \% \mathrm{~A}$ in $10 \mathrm{~min}$, and held for 5 $\min$.

In addition, HPLC-UV (UV absorbance detector, $214 \mathrm{~nm}$ ) was optimized for TBBPA BDBPE, because the poor sensitivity of HPLC-APCI-MS/MS for this analyte (see the Results and Discussion). The C18 column $(150 \times 4.6 \mathrm{~mm}, 5 \mu \mathrm{m}$ particle size, Sunfire) was used, and HPLC separation was carried out with an initial mobile phase setting of $90 \%$ acetonitrile (A) and $10 \%$ water (B) and a linear gradient elution from 90 to $100 \% \mathrm{~A}$ over a $20 \mathrm{~min}$ period, followed by an isocratic hold for $5 \mathrm{~min}$ at $100 \%$ A. For evaluation of instrumental detection limits (IDLs, 3 times the signal-to-noise ratio) and standard curve for quantitative analysis, a series of different concentrations of target chemicals were injected into the analytical instrument.

Sample Preparation. Soil $(1 \mathrm{~g})$, sediment $(1 \mathrm{~g})$, rice hull $(0.25 \mathrm{~g})$, and earthworm or mollusks $(0.25 \mathrm{~g})$ samples were mixed with $15 \mathrm{~g}$ of anhydrous $\mathrm{Na}_{2} \mathrm{SO}_{4}$ and extracted with 50 $\mathrm{mL}$ of $\mathrm{DCM} / n$-hexane $(1: 1, \mathrm{v} / \mathrm{v})$ at $100{ }^{\circ} \mathrm{C}$ and 1500 psi using accelerated solvent extraction for $12 \mathrm{~min}$ with 3 cycles (ASE 300, Dionex). The extract was rotary-evaporated and concentrated to $5 \mathrm{~mL}$ under a nitrogen flow. Then, the extract was cleaned up by an automatic GPC for primary cleanup. The eluate was rotary-evaporated and concentrated to incipient dryness under a gentle nitrogen flow and solvent-exchanged with $1 \mathrm{~mL}$ of $n$-hexane. Then, the samples were added to a SPE cartridge [Bakerbond, $6 \mathrm{~mL}, 500 \mathrm{mg}$ of silica ( $\mathrm{SiOH}$ ) absorbents, Mallinckrodt Baker, Inc., Germany], preconditioned with $3 \mathrm{~mL}$ of DCM and $6 \mathrm{~mL}$ of $n$-hexane. The cartridge was washed with $6 \mathrm{~mL}$ of DCM/n-hexane $(1: 9, \mathrm{v} / \mathrm{v})$ to remove impurities. To evaluate the recovery performance, sediment, soil, and mollusk samples that were determined to not contain the analytes were spiked with the target compounds and underwent the whole pretreatment and analytical procedure (see the Supporting Information for details).

\section{RESULTS AND DISCUSSION}

Identification of TBBPA AE and TBBPA DBPE. Because no commercial standards are available for TBBPA $\mathrm{AE}$ and TBBPA DBPE, these two target chemicals were synthesized and characterized. Nuclear magnetic resonance (NMR) results indicated that high purity was reached for both compounds (see Figures S3 and S4 of the Supporting Information). The purity of TBBPA AE and TBBPA DBPE was 98.0 and $97.9 \%$ according to the results from HPLC-UV $(214 \mathrm{~nm}$ ) (data not shown). HPLC-APCI-Q-TOF-MS was further used for identification and to obtain detailed mass spectra for TBBPA $\mathrm{AE}$ and TBBPA DBPE. The full-scan mass spectrum $(\mathrm{m} / \mathrm{z}$ 450-800) of TBBPA AE using HPLC-Q-TOF-MS can be seen in Figure 1A. The ions at $m / z 582.970$ were the dominant ion clusters containing four bromine atoms with the theoretical isotope distribution value $(1: 4: 6: 4: 1)$. The cluster ions at $\mathrm{m} / z$ 541.632 with very low intensity could be explained by the ion cluster $\left[\mathrm{M}-\mathrm{C}_{3} \mathrm{H}_{5}-\mathrm{H}\right]^{-}$because of the breaking of the ether bond. For TBBPA DBPE identification, the ion clusters $[\mathrm{M}-$ $\mathrm{H}]^{-}$centered at 742.726 with isotopic $\mathrm{Br}$ distribution 

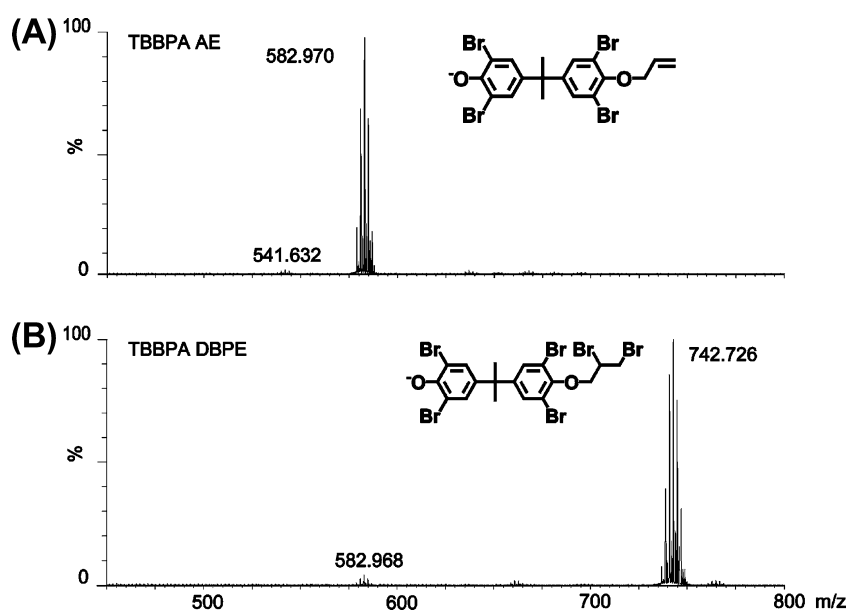

Figure 1. Full scan of TOF mass spectra of (A) TBBPA AE and (B) TBBPA DBPE.

demonstrated the presence of six bromine atoms (Figure 1B), while the minor cluster around 582.968 might be ions $[\mathrm{M}-$ $\left.\mathrm{C}_{3} \mathrm{H}_{5}\right]^{-}$generated by TBBPA $\mathrm{AE}$ as impurity. Both of the TBBPA derivatives tend to undergo phenolic hydroxyl ionization and form the main ions $[\mathrm{M}-\mathrm{H}]^{-}$, which is similar to ionization of TBBPA under ESI mode. ${ }^{24,25}$ The other lesser ion fragments cannot be explained by TBBPA AE or TBBPA DBPE but might be due to the ionization of minor impurities. The results show that the two target chemicals were wellidentified using NMR, HPLC-UV, and Q-TOF-MS. The high purity of TBBPA AE and TBBPA DBPE could therefore be used as a standard for the identification and quantification of these chemicals in this study.

Quantitative Analysis. To quantify TBBPA, TBBPA AE, TBBPA BAE, TBBPA DBPE, and TBBPA BDBPE, both HPLC-APCI-MS/MS and HPLC-ESI-MS/MS methods were optimized to determine the best ionization mode. Under APCI mode, for Q1 scan, the precursor ions of TBBPA AE and TBBPA DBPE were at $m / z 582.9\left([\mathrm{M}-\mathrm{H}]^{-}\right)$and $m / z 742.7$ $\left([\mathrm{M}-\mathrm{H}]^{-}\right)$, respectively, which were the same as the precursor ions of TBBPA BAE $(m / z 582.9)\left(\left[\mathrm{M}-\mathrm{C}_{3} \mathrm{H}_{5}\right]^{-}\right)$ and TBBPA BDBPE $\left(m / z\right.$ 742.7) $\left(\left[\mathrm{M}-\mathrm{C}_{3} \mathrm{H}_{3} \mathrm{Br}_{2}\right]^{-}\right)$because of the breaking of the ether bond (see Figure S5 of the Supporting Information). Neither TBBPA AE nor TBBPA DBPE yielded $\mathrm{Br}^{-}$, which is different from that of other BFRs, such as polybrominated diphenyl ethers (PBDEs) and hydroxylated PBDE under MRM mode. ${ }^{26,27}$ Thus, the ions at $\mathrm{m} / z 582.9$ and 742.7 were chosen as the precursor ions (see Table S1 of the Supporting Information). For the second ionization, because of the breaking of the ether bond, the precursor ions of TBBPA AE and TBBPA BAE could form the product ions $(m / z 526.5)$ (see Table $S 1$ of the Supporting Information), which were also similar to the product ions of TBBPA AE and TBBPA BDBPE because of the same ionization mechanism (see Figure S5 of the Supporting Information). Thus, all of the product ions of TBBPA AE/TBBPA BAE and TBBPA DBPE/TBBPA BDBPE were $\left[\mathrm{M}-\mathrm{C}_{3} \mathrm{H}_{5}-\mathrm{CH}_{3}\right]^{-}$ $(\mathrm{m} / z$ 526.5), which was found to be the most intensive $\mathrm{m} / z$ and was sufficiently stable to be selected as the product ion for MRM quantification for the four chemicals (see Table S1 and Figure S5 of the Supporting Information). For TBBPA AE and TBBPA DBPE analysis, the sensitivity using ESI mode was found to be higher than that of the APCI-based method (Table 1). Thus, the HPLC-ESI-MS/MS method was used to determine the concentration of TBBPA, TBBPA AE, and TBBPA DBPE (see Figure S6 of the Supporting Information). However, the ESI mode did not produce any significant precursor ions for TBBPA BAE and TBBPA BDBPE under fullscan optimization, which is different from another ESI-based method for TBBPA BAE analysis. ${ }^{28}$ HPLC-APCI-MS/MS was therefore used to analyze TBBPA BAE in environmental samples, as previously described (see Figure S7 of the Supporting Information). ${ }^{15}$ Although the specific spectrum of TBBPA BDBPE could be obtained (see Table S1 and Figure S5 of the Supporting Information), the sensitivity was not enough to determine its concentration in real environmental samples. Thus, we selected HPLC coupled with an UV detector (214 $\mathrm{nm}$ absorption) to determine TBBPA BDBPE in the environmental samples (Table 1 and Figure $S 8$ of the Supporting Information) Using the HPLC-UV method, almost no matrix effect was found, which suggested that the HPLC-UV signal for TBBPA BDBPE was specific enough for analysis.

For sample pretreatment, the SPE cleanup method was separately optimized for the biotic and abiotic samples (see the Supporting Information). For soil and sediment samples, the five target chemicals could be eluted satisfactorily using a mixture of $\mathrm{DCM} / n$-hexane $(7: 3, \mathrm{v} / \mathrm{v})$ and the recovery was 76 $\pm 15 \%$ (TBBPA), $89 \pm 14 \%$ (TBBPA AE), $75 \pm 10 \%$ (TBBPA

Table 1. Recovery, IDLs, Method LODs, and LOQs of the Four Target Chemicals Using HPLC-APCI-MS/MS or HPLCESI-MS/MS

\begin{tabular}{|c|c|c|c|c|c|c|c|c|}
\hline & \multirow[b]{2}{*}{ compounds } & \multirow[b]{2}{*}{$\begin{array}{l}\text { method recovery } \\
\qquad \%) \pm \mathrm{SD}^{a}\end{array}$} & \multicolumn{3}{|c|}{ APCI } & \multicolumn{3}{|c|}{ ESI } \\
\hline & & & $\begin{array}{l}\mathrm{IDL} \\
(\mathrm{pg})\end{array}$ & $\begin{array}{l}\mathrm{LOD} \\
(\mathrm{pg} / \mathrm{g})\end{array}$ & $\begin{array}{l}\mathrm{LOQ} \\
(\mathrm{pg} / \mathrm{g})\end{array}$ & $\begin{array}{l}\mathrm{IDL} \\
(\mathrm{pg})\end{array}$ & $\begin{array}{l}\mathrm{LOD} \\
(\mathrm{pg} / \mathrm{g})\end{array}$ & $\begin{array}{l}\mathrm{LOQ} \\
(\mathrm{pg} / \mathrm{g})\end{array}$ \\
\hline \multirow{4}{*}{$\begin{array}{l}\text { mollusk, earthworm, and rice } \\
\text { hull }\end{array}$} & TBBPA AE & $78 \pm 13$ & 0.04 & 10.0 & 40.0 & 0.03 & 3.0 & 10.0 \\
\hline & TBBPA DBPE & $84 \pm 9$ & 0.2 & 20.0 & 73.0 & 0.04 & 8.0 & 32.0 \\
\hline & TBBPA BAE & $85 \pm 19$ & 130 & 20000 & 80000 & & & \\
\hline & TВBPA BDBPE $^{b}$ & $81 \pm 17$ & 800 & 50000 & 180000 & & & \\
\hline \multirow[t]{5}{*}{ soil and sediment } & TBBPA & $76 \pm 15$ & 2.0 & 50.0 & 180.0 & 0.4 & 30 & 70 \\
\hline & TBBPA AE & $89 \pm 14$ & 0.04 & 5.0 & 10.0 & 0.02 & 1.5 & 4.0 \\
\hline & TBBPA DBPE & $75 \pm 10$ & 0.2 & 10 & 24 & 0.04 & 4.0 & 5.0 \\
\hline & ТВВРА ВАE & $85 \pm 9$ & 130 & 5000 & 1500 & & & \\
\hline & ТВBРA ВDВРЕ $^{b}$ & $73 \pm 17$ & 800 & 25000 & 60000 & & & \\
\hline
\end{tabular}

${ }^{a}$ The method recovery was evaluated on the basis of spiked matrix samples $(n=3)$ (see the Supporting Information). ${ }^{b}$ The IDL, LOD, and LOQ of TBBPA BDBPE described in the table were based on HPLC-UV because of the poor sensitivity of the HPLC-MS/MS method. 

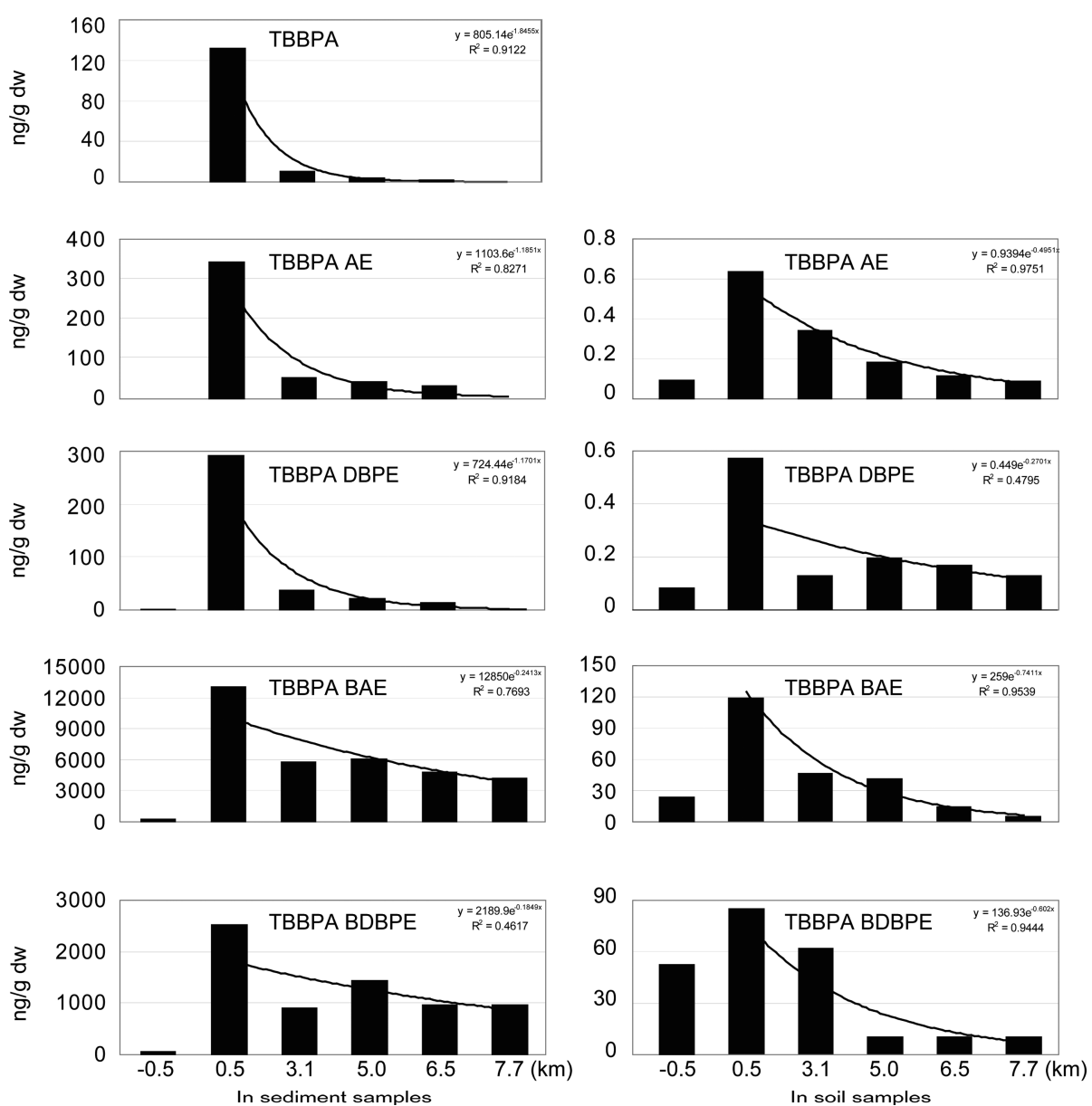

Figure 2. Concentrations of TBBPA, TBBPA AE, TBBPA DBPE, TBBPA BAE, and TBBPA BDBPE in sediment and soil samples collected at an increasing distance from the manufacturing plant.

DBPE), $85 \pm 9 \%$ (TBBPA BAE), and $73 \pm 17 \%$ (TBBPA $\mathrm{BDBPE})$. However for biological samples, the mixture of $\mathrm{DCM} / n$-hexane $(7: 3, \mathrm{v} / \mathrm{v})$ showed a matrix effect during HPLC-MS/MS analysis. By changing the mixture to DCM $/ n$ hexane $(1: 1, \mathrm{v} / \mathrm{v})$, the satisfactory recoveries were obtained for TBBPA AE, TBBPA DBPE, TBBPA BAE, and TBBPA BDBPE $(78 \pm 13,84 \pm 9,85 \pm 19$, and $81 \pm 17 \%$, respectively). However, the recovery for TBBPA was lower than $50 \%$. Thus, for biological samples, a mixture of DCM/n-hexane $(1: 1, \mathrm{v} / \mathrm{v})$ was used to elute chemicals and TBBPA AE, TBBPA DBPE, TBBPA BAE, and TBBPA BDBPE were analyzed.

TBBPA AE/TBBPA DBPE in Technical Products. To determine whether or not TBBPA BAE and TBBPA BDBPE technical products also contain TBBPA AE and TBBPA DBPE as byproducts during manufacturing, technical products of TBBPA BAE and TBBPA BDBPE in powder form were purchased from the same manufacturing plant (see Figure $S 1$ of the Supporting Information) located in Liuyang and analyzed with our developed method. It was found that TBBPA AE and TBBPA DBPE could be positively detected in the technical products at concentrations of 0.48 and $0.62 \mathrm{mg} / \mathrm{g}$, respectively. This result indicated that TBBPA AE and TBBPA DBPE could also be formed during production of the other TBBPA derivatives, and thus, the manufacturing plant could be a source of TBBPA AE and TBBPA DBPE in the environment. Levels of TBBPA in TBBPA BAE and TBBPA BDBPE technical products were lower than the limit of detection
(LOD) $(<0.03 \mathrm{ng} / \mathrm{g})$, suggesting nearly no or a relatively low contamination of TBBPA in the technical products.

TBBPA AE/TBBPA DBPE and TBBPA BAE/TBBPA BDBPE in the Ambient Environment near the BFR Manufacturing Plant. Sediment and soil samples were collected to investigate the distribution characteristics of the five chemicals near the manufacturing plant (see Figure S1 of the Supporting Information). The concentrations of the five chemicals in the environmental matrices are shown in Figure 2. The highest levels of the five chemicals were detected in sediment samples collected near the outlet. Low sediment levels were found at the upstream site, whereas elevated levels were found in sediment $500 \mathrm{~m}$ downstream of the plant, which indicated that the plant is most likely the release source of the TBBPA derivatives. The concentration of TBBPA BAE in sediment near the outlet was about $13 \mu \mathrm{g} / \mathrm{g}$, which was higher than that of TBBPA BDBPE at $2.5 \mu \mathrm{g} / \mathrm{g}$. The levels of TBBPA AE (1.0-346.6 ng/g) and TBBPA DBPE (0.7-292.7 ng/g) were much lower than those of TBBPA BAE and TBBPA $\mathrm{BDBPE}$, with higher average levels of TBBPA AE compared to TBBPA DBPE. The level of TBBPA was the lowest among the five chemicals. TBBPA was detected the highest near the outlet $(132 \mathrm{ng} / \mathrm{g}$ ) and rapidly decreased to below the limit of quantification (LOQ) $(0.03 \mathrm{ng} / \mathrm{g})$ at $7 \mathrm{~km}$.

The exponential declining trends of TBBPA with a distance from the plant were more rapid than TBBPA AE and TBBPA DBPE (Figure 2). Similarly, the decreasing trends of TBBPA $\mathrm{AE}$ and TBBPA DBPE concentrations were more obvious than 

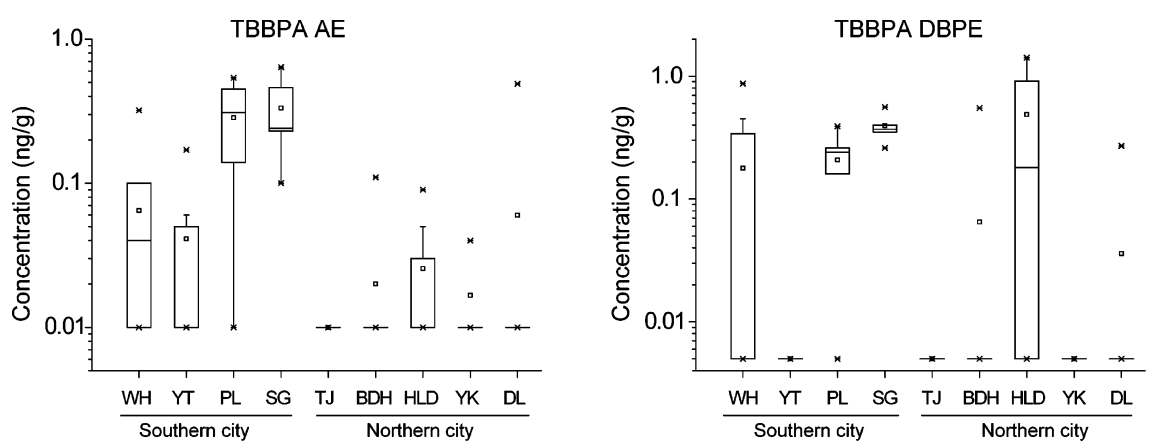

Figure 3. Concentrations of TBBPA AE and TBBPA DBPE in mollusks around the Bohai region. Concentrations are shown in the logarithmic $y$ scale. The box represents the extent of 25 th and 75 th percentiles, and the middle line represents the median value. Mean values are symbolized by " $\square$ ". The whiskers extending from the box show the lowest and highest non-outlier values. " $¥$ " represents the lowest and highest values for each chemical among all samples in each city.

TBBPA BAE and TBBPA BDBPE, reducing to about 1 order of magnitude at $3 \mathrm{~km}$ downstream and approximately 2 orders of magnitude at around $8 \mathrm{~km}$ (Figure 2). The more rapid decreasing levels of TBBPA AE and TBBPA DBPE compared to TBBPA BAE and TBBPA BDBPE along the river flow direction could indicate a lower sorption capacity or higher degradation rate of the former compounds, and a similar trend was also found for TBBPA compared to TBBPA AE and TBBPA DBPE (see panels A and B of Figure S9 of the Supporting Information). The U.S. Estimation Programs Interface (EPI) suite models is often used for estimating the physicochemical properties of chemicals using descriptors, such as empirical atom/fragment/group/bond contribution. ${ }^{16,29,30}$ In the current study, EPI version 4.1 was used to predict the atmospheric oxidation half-life $\left(\mathrm{AO}_{1 / 2}\right), \log K_{\mathrm{aw}}, \log K_{\mathrm{ow}}$, and $\log K_{\mathrm{oa}}$. The $\log K_{\mathrm{ow}}$ value of TBBPA (7.21) is lower than TBBPA AE (8.61) and TBBPA DBPE (9.36), which are subsequently lower than TBBPA BAE (10.02) and TBBPA BDBPE (11.52) (see Table S2 of the Supporting Information). The ratio of TBBPA AE/TBBPA BAE and TBBPA DBPE/ TBBPA BDBPE in the sediment near the outlet was 2.6 and $11.5 \%$ (see Figure S9A of the Supporting Information), respectively, and was significantly higher than that in the technical products $(<0.1 \%)$. This suggested that the byproducts might be formed along with the production of TBBPA BAE and TBBPA BDBPE during wastewater treatment processes or environmental degradation of TBBPA BAE/TBBPA BDBPE.

Levels of TBBPA AE and TBBPA DBPE in soil ranged from 0.09 to $0.64 \mathrm{ng} / \mathrm{g}$ and from 0.13 to $0.58 \mathrm{ng} / \mathrm{g}$, respectively, distinctly lower than those in the sediment, and the exponential decreasing trends also suggested that they were mainly released from the plant (Figure 2). The levels of TBBPA were all lower than the LOD $(<0.03 \mathrm{ng} / \mathrm{g})$. The soil contamination could be attributed to atmospheric deposition as well as the river water turbulence and volatilization. Soil concentrations of TBBPA BAE and TBBPA BDBPE ranged from below the LOD $(<5 \mathrm{ng} /$ g) to $24.1 \mathrm{ng} / \mathrm{g}$ and from below the LOD $(<25 \mathrm{ng} / \mathrm{g})$ to 85.3 $\mathrm{ng} / \mathrm{g}$, respectively, which were higher than for TBBPA AE and TBBPA DBPE. The ratio of TBBPA AE and TBBPA DBPE versus TBBPA BAE and TBBPA BDBPE both increased with the distance (see Figure S9C of the Supporting Information). This might be due to the differences in physical-chemical properties (see Table S2 of the Supporting Information) and degradation processes, such as biological transformation (see Figure S10 of the Supporting Information). ${ }^{4}$
The decreasing trends of TBBPA AE and TBBPA DBPE were also found for rice hull and earthworm. The levels of TBBPA AE and TBBPA DBPE were at the same order of magnitude as those detected in soil samples (see Figure S11 of the Supporting Information). For TBBPA AE, the rank of the average concentrations among the matrices was rice hull $(0.45$ $\mathrm{ng} / \mathrm{g})>$ soil $(0.32 \mathrm{ng} / \mathrm{g})>$ earthworm $(0.30 \mathrm{ng} / \mathrm{g})$, whereas the rank of the average concentrations of TBBPA DBPE was earthworm $(0.46 \mathrm{ng} / \mathrm{g})>$ rice hull $(0.27 \mathrm{ng} / \mathrm{g})>$ soil $(0.26 \mathrm{ng} /$ g).

These results together indicated that TBBPA BAE and TBBPA BDBPE production could result in related contamination to the surrounding environment. TBBPA $A E$ and TBBPA DBPE were shown to most likely be released from the manufacturing plant. Furthermore, positive detection in rice hull and worms suggested a potential exposure risks to surrounding biota because these contaminants might enter the local food chains.

TBBPA AE/TBBPA DBPE and TBBPA BAE/TBBPA BDBPE in Mollusks around Bohai Sea of China. To investigate whether TBBPA AE/TBBPA BAE and TBBPA DBPE/TBBPA BDBPE could be present in the marine environment, we analyzed mollusks collected from the Bohai Sea in China (see Figure S1 of the Supporting Information). The concentrations in the soft tissue of different mollusk species in each city are shown in Figure 3. TBBPA BAE and TBBPA BDBPE in all mollusk samples were below the LOQ ( $<20 \mathrm{ng} / \mathrm{g}$ for TBBPA BAE and $<50 \mathrm{ng} / \mathrm{g}$ for TBBPA BDBPE). The concentrations of TBBPA BAE and TBBPA BDBPE in mollusks might be low because of their poor solubility, high log $K_{\text {ow }}$, and generally low release amounts. Although this current method for TBBPA BAE and TBBPA BDBPE could be used to determine the concentration around the manufacturing plant, a trace-level determination of these chemicals is not feasible. A more sensitive method could probably be developed using APPI as the ionization source, but this equipment is unfortunately not available in our lab. However, the detection frequencies of TBBPA AE and TBBPA DBPE were 45 and $32 \%$, respectively, which were lower than those for tris $(2,3-$ dibromopropyl) isocyanurate (TBC, 77\%), HBCD, (99\%), and PBDEs $(100 \%)$ that were determined in the same set of samples in a previous study. ${ }^{30}$ TBBPA AE concentrations ranged from below the LOD $(<0.003 \mathrm{ng} / \mathrm{g})$ to $0.54 \mathrm{ng} / \mathrm{g}$, with an average of $0.09 \mathrm{ng} / \mathrm{g}$. The average concentration of TBBPA DBPE was $0.15 \mathrm{ng} / \mathrm{g}$ and ranged from below the LOQ $(<0.008$ $\mathrm{ng} / \mathrm{g}$ ) to $1.41 \mathrm{ng} / \mathrm{g}$. For convenience in the discussion, the 
sampling sites were divided into southern and northern Bohai regions (see Figure S1 of the Supporting Information and Figure 3). Previous results showed that the highest concentration of PBDEs and HBCD was found in Tianjin, ${ }^{30}$ but TBBPA AE and TBBPA DBPE were not detected in this city (Figure 3). Levels of PBDEs and HBCD were not found to be significantly different between southern and northern sites. In contrast, TBBPA AE was predominantly distributed in regions around the south of Bohai Sea (average concentration of $0.17 \mathrm{ng} / \mathrm{g}$ with a detection frequency of $76 \%$ ), significantly higher than those found in northern cities (average concentration of $0.03 \mathrm{ng} / \mathrm{g}$ with a detection frequency of $13 \%)$. The highest level of TBBPA DBPE was found in Huludao, but there was in general no significant difference between southern and northern cities, although the detection frequencies were higher in southern cities $(51 \%$ in southern cities versus $21 \%$ in northern cities) (Figure 3 ). In the southern sites, the concentrations of TBBPA AE and TBBPA DBPE were similar, showing the highest levels in Penglai and Shouguang (Figure 3), which indicated that some point sources, such as BFR manufacturing plants around southern Bohai Bay, may influence the distribution of TBBPA AE and TBBPA DBPE. For instance, several BFR manufacturing plant has been documented that companies including Shouguang Guangda Chemicals (Shouguang, Shandong, China, http://www. sggdchem.com), Shou Guang Longfa Chemicals (Qindao, Shandong, China, http://www.longfachem.com), Weidong International Group, Ltd. (Weifang, Shandong, China, http:// www.oceanchemical.en.ecplaza.net), and Zouping Mingxing Chemical Co., Ltd. (Liangzou, Shandong, China, http://www. zoutong.com.cn) produce TBBPA BAE or TBBPA BDBPE. ${ }^{4}$

There was no significant correlation between TBBPA AE and TBBPA DBPE for samples that were positively detected for both compounds (data not shown; $n=19$ ), suggesting different sources or environmental fates between these two compounds in the marine environment. The concentrations grouped by each species were shown in Tables S3 and S4 of the Supporting Information.

Occurrence of TBBPA Derivatives, Byproducts, or Degradation Products in the Environment. Several studies have reported the presence of some novel or emerging BFRs in various environmental compartments. ${ }^{4,15,31-33}$ The identification of TBBPA AE and TBBPA DBPE in this study adds further information to the list of newly detected brominated organic contaminants in the environment. TBBPA has been listed as one of the top priority chemicals in a screening study based on their high production volume and properties, such as $\log K_{\mathrm{ow}}$ and environmental persistency. ${ }^{16}$ TBBPA AE and TBBPA DBPE might also be potentially bioaccumulative because of $\log K_{\text {ow }}$ values $>5$ (EPA Suite; see Table S2 of the Supporting Information). ${ }^{29}$ Among the five chemicals, the predicted air-water partitioning constants $\left(\log K_{\mathrm{aw}}\right)$ were lower than -8 , except for TBBPA BAE (see Table S2 of the Supporting Information). This suggests their tendency to partition to water, which is different from, e.g., pentaBDE, which is comparatively more prone to be evaporated to the atmosphere.

To investigate whether TBBPA could be one of the transformation products of the TBBPA derivatives, the University of Minnesota Pathway Prediction System (UMPPS) was used to calculate the potential microbial catabolic reaction of TBBPA BAE and TBBPA BDBPE. TBBPA BAE tends to form TBBPA $A E$ and TBBPA in sequence as the result of the microbial transformation reaction (bt0023) (see Figure S12 of the Supporting Information). ${ }^{34}$ Similarly, TBBPA BDBPE could degrade to TBBPA DBPE and TBBPA in a similar pathway. Therefore, the results indicate that microbial transformation of TBBPA BAE and TBBPA BDBPE could be one of the sources of TBBPA AE and TBBPA DBPE in the environment. The sensitive method that we report in this study for three TBBPA derivatives could be adapted for the analysis of other complex abiotic and biotic environmental matrices from other regions around the world.

\section{ASSOCIATED CONTENT}

\section{Supporting Information}

Additional information as noted in the text (recovery evaluation, Tables S1-S4, and Figures S1-S12). This material is available free of charge via the Internet at http://pubs.acs.org.

\section{AUTHOR INFORMATION}

\section{Corresponding Author}

*Telephone/Fax: +86-10-62849179. E-mail: jbshi@rcees.ac.cn (J.S.); sjliu@rcees.ac.cn (S.L.).

\section{Notes}

The authors declare no competing financial interest.

\section{ACKNOWLEDGMENTS}

The work described here was supported by the National Basic Research Program of China (2009CB421605) and the National Natural Science Foundation of China (20890111, 20921063, and 20977107).

\section{REFERENCES}

(1) Hites, R. A. Polybrominated diphenyl ethers in the environment and in people: A meta-analysis of concentrations. Environ. Sci. Technol. 2004, 38 (4), 945-956.

(2) Hamers, T.; Kamstra, J. H.; Sonneveld, E.; Murk, A. J.; Kester, M. H. A.; Andersson, P. L.; Legler, J.; Brouwer, A. In vitro profiling of the endocrine-disrupting potency of brominated flame retardants. Toxicol. Sci. 2006, 92 (1), 157-173.

(3) Schecter, A.; Pavuk, M.; Päpke, O.; Ryan, J. J.; Birnbaum, L.; Rosen, R. Polybrominated diphenyl ethers (PBDEs) in US mothers' milk. Environ. Health Perspect. 2003, 111 (14), 1723-1729.

(4) Covaci, A.; Harrad, S.; Abdallah, M. A. E.; Ali, N.; Law, R. J.; Herzke, D.; de Wit, C. A. Novel brominated flame retardants: A review of their analysis, environmental fate and behaviour. Environ. Int. 2011, 37 (2), 532-556

(5) Söderstrom, G.; Sellström, U.; De Wit, C. A.; Tysklind, M. Photolytic debromination of decabromodiphenyl ether (BDE 209). Environ. Sci. Technol. 2004, 38 (1), 127-132.

(6) Stapleton, H. M.; Brazil, B.; Holbrook, R. D.; Mitchelmore, C. L.; Benedict, R.; Konstantinov, A.; Potter, D. In vivo and in vitro debromination of decabromodiphenyl ether (BDE 209) by juvenile rainbow trout and common carp. Environ. Sci. Technol. 2006, 40 (15), $4653-4658$.

(7) Hakk, H.; Letcher, R. J. Metabolism in the toxicokinetics and fate of brominated flame retardants-A review. Environ. Int. 2003, 29 (6), $801-828$.

(8) von der Recke, R.; Vetter, W. Synthesis and characterization of 2,3-dibromopropyl-2,4,6-tribromophenyl ether (DPTE) and structurally related compounds evidenced in seal blubber and brain. Environ. Sci. Technol. 2007, 41 (5), 1590-1595.

(9) Law, R. J. Tetrabromobisphenol A: Investigating the worst-case scenario. Mar. Pollut. Bull. 2009, 58 (4), 459-460.

(10) Covaci, A.; Voorspoels, S.; Abdallah, M. A. E.; Geens, T.; Harrad, S.; Law, R. J. Analytical and environmental aspects of the 
flame retardant tetrabromobisphenol-A and its derivatives. J. Chromatogr., A 2009, 1216 (3), 346-363.

(11) Bergman, A.; Ryden, A.; Law, R. J.; de Boer, J.; Covaci, A.; Alaee, M.; Birnbaum, L.; Petreas, M.; Rose, M.; Sakai, S.; Van den Eede, N.; van der Veen, I. A novel abbreviation standard for organobromine, organochlorine and organophosphorus flame retardants and some characteristics of the chemicals. Environ. Int. 2012, 49, $57-82$.

(12) Morris, S.; Allchin, C. R.; Zegers, B. N.; Haftka, J. J. H.; Boon, J. P.; Belpaire, C.; Leonards, P. E. G.; Van Leeuwen, S. P. J.; De Boer, J. Distributon and fate of HBCD and TBBPA brominated flame retardants in north sea estuaries and aquatic food webs. Environ. Sci. Technol. 2004, 38 (21), 5497-5504.

(13) Köppen, R.; Becker, R.; Jung, C.; Piechotta, C.; Nehls, I. Investigation of extraction procedures and HPLC-DAD/MS for the determination of the brominated flame retardant tetrabromobisphenol A bis(2,3-dibromopropylether) in environmental samples. Anal. Bioanal. Chem. 2006, 384 (7-8), 1485-1492.

(14) Letcher, R. J.; Chu, S. G. High-sensitivity method for determination of tetrabromobisphenol-S and tetrabromobisphenol-A derivative flame retardants in Great Lakes herring gull eggs by liquid chromatography-atmospheric pressure photoionization-tandem mass spectrometry. Environ. Sci. Technol. 2010, 44 (22), 8615-8621.

(15) Qu, G. B.; Shi, J. B.; Wang, T.; Fu, J. J.; Li, Z. N.; Wang, P.; Ruan, T.; Jiang, G. B. Identification of tetrabromobisphenol A diallyl ether as an emerging neurotoxicant in environmental samples by bioassay-directed fractionation and HPLC-APCI-MS/MS. Environ. Sci. Technol. 2011, 45 (11), 5009-5016.

(16) Howard, P. H.; Muir, D. C. G. Identifying new persistent and bioaccumulative organics among chemicals in commerce. Environ. Sci. Technol. 2010, 44 (7), 2277-2285.

(17) European Commission Joint Research Centre (JRC). Summary Risk Assessment Report on TBBP-A; JRC: Ispra, Italy, 2006.

(18) United States Environmental Protection Agency (U.S. EPA). Non-confidential 2006 IUR Records by Chemical, Including Manufacturing, Processing and Use Information; U.S. EPA: Washington, D.C., 2006; http://cfpub.epa.gov/iursearch/2006 iur natlcheminfo.cfm?id=313.

(19) Xiao, J. A Perspective on the Development of Brominated Flame Retardants in China, 2006; http://www.polymer.cn/html/ industrynews/200612/15/2007529102655763.htm.

(20) Fu, J. J.; Wang, T.; Wang, P.; Qu, G. B.; Wang, Y. W.; Zhang, Q. H.; Zhang, A. Q.; Jiang, G. B. Temporal trends (2005-2009) of $\mathrm{PCDD} / \mathrm{Fs}$, PCBs, PBDEs in rice hulls from an e-waste dismantling area after stricter environmental regulations. Chemosphere 2012, 88 (3), 330-335.

(21) Sellström, U.; De Wit, C. A.; Lundgren, N.; Tysklind, M. Effect of sewage-sludge application on concentrations of higher-brominated diphenyl ethers in soils and earthworms. Environ. Sci. Technol. 2005, 39 (23), 9064-9070.

(22) Isobe, T.; Takada, H.; Kanai, M.; Tsutsumi, S.; Isobe, K. O.; Boonyatumanond, R.; Zakaria, M. P. Distribution of polycyclic aromatic hydrocarbons (PAHs) and phenolic endocrine disrupting chemicals in south and southeast Asian mussels. Environ. Monit. Assess. 2007, 135 (1-3), 423-440.

(23) Wang, Y. W.; Wang, T.; Li, A.; Fu, J. J.; Wang, P.; Zhang, Q. H.; Jiang, G. B. Selection of bioindicators of polybrominated diphenyl ethers, polychlorinated biphenyls, and organochlorine pesticides in mollusks in the Chinese Bohai Sea. Environ. Sci. Technol. 2008, 42 (19), 7159-7165.

(24) Saint-Louis, R.; Pelletier, E. LC-ESI-MS-MS method for the analysis of tetrabromobisphenol $\mathrm{A}$ in sediment and sewage sludge. Analyst 2004, 129 (8), 724-730.

(25) Chu, S. G.; Haffner, G. D.; Letcher, R. J. Simultaneous determination of tetrabromobisphenol $\mathrm{A}$, tetrachlorobisphenol $\mathrm{A}$, bisphenol $\mathrm{A}$ and other halogenated analogues in sediment and sludge by high performance liquid chromatography-electrospray tandem mass spectrometry. J. Chromatogr., A 2005, 1097 (1-2), 25-32.

(26) Kato, Y.; Okada, S.; Atobe, K.; Endo, T.; Matsubara, F.; Oguma, T.; Haraguchi, K. Simultaneous determination by APCI-LC/MS/MS of hydroxylated and methoxylated polybrominated diphenyl ethers found in marine biota. Anal. Chem. 2009, 81 (14), 5942-5948.

(27) Abdallah, M. A.; Harrad, S.; Covaci, A. Isotope dilution method for determination of polybrominated diphenyl ethers using liquid chromatography coupled to negative ioonization atmospheric pressure photoionization tandem mass spectrometry: Validation and application to house dust. Anal. Chem. 2009, 81 (17), 7460-7467.

(28) Ismail, N.; Pleskach, K.; Marvin, C. H.; Whittle, D. M.; Keir, M.; Helm, P. A.; Tomy, G. T. Temporal trends of flame retardants in Lake Ontario lake trout (1979-2004). Organohalogen Compd. 2006, 68, $1808-1811$.

(29) United States Environmental Protection Agency (U.S. EPA). Estimation Programs Interface (EPI) Suite v4.10; U.S. EPA: Washington, D.C., Oct 2011; http://www.epa.gov/oppt/exposure/pubs/episuitedl. htm.

(30) Zhu, N. L.; Li, A.; Wang, T.; Wang, P.; Qu, G. B.; Ruan, T.; Fu, J. J.; Yuan, B.; Zeng, L. X.; Wang, Y. W.; Jiang, G. B. Tris(2,3dibromopropyl) isocyanurate, hexabromocyclododecanes, and polybrominated diphenyl ethers in mollusks from Chinese Bohai Sea. Environ. Sci. Technol. 2012, 46 (13), 7174-7181.

(31) Hoh, E.; Zhu, L. Y.; Hites, R. A. Novel flame retardants, 1,2bis(2,4,6-tribromophenoxy)ethane and 2,3,4,5,6-pentabromoethylbenzene, in United States' environmental samples. Environ. Sci. Technol. 2005, 39 (8), 2472-2477.

(32) Tomy, G. T.; Pleskach, K.; Arsenault, G.; Potter, D.; Mccrindle, R.; Marvin, C. H.; Sverko, E.; Tittlemier, S. Identification of the novel cycloaliphatic brominated flame retardant 1,2-dibromo-4-(1,2dibromoethyl)cyclo-hexane in Canadian arctic beluga (Delphinapterus leucas). Environ. Sci. Technol. 2008, 42 (2), 543-549.

(33) Gauthier, L. T.; Hebert, C. E.; Weseloh, D. V. C.; Letcher, R. J. Current-use flame retardants in the eggs of herring gulls (Larus argentatus) from the Laurentian Great Lakes. Environ. Sci. Technol. 2007, 41 (13), 4561-4567.

(34) Ng, C. A.; Scheringer, M.; Fenner, K.; Hungerbühler, K. A framework for evaluating the contribution of transformation products to chemical persistence in the environment. Environ. Sci. Technol. 2011, 45 (1), 111-117. 